\title{
Dielectric relaxation process of a partially unwound helical structure in ferroelectric liquid crystals
}

\author{
Amit Choudhary, ${ }^{1}$ Ambika Bawa, ${ }^{2,3}$ Rajesh, ${ }^{2,3}$ Surinder P. Singh, ${ }^{2}$ and Ashok M. Biradar ${ }^{2,3, *}$ \\ ${ }^{1}$ Physics Department, Deshbandhu College (University of Delhi, Delhi), Kalkaji, New Delhi 110019, India \\ ${ }^{2}$ CSIR-Academy of Scientific and Innovative Research (AcSIR), Taramani, Chennai 600113, India \\ ${ }^{3}$ CSIR-National Physical Laboratory, Dr. K. S. Krishnan Marg, New Delhi 110012, India
}

(Received 26 December 2016; published 8 June 2017)

\begin{abstract}
The fluctuations of unwound helical structure have been observed in deformed helix ferroelectric liquid crystal (DHFLC) and conventional FLC sample cells. The helix is partially unwound by strong anchoring on the substrates. In such sample cells, the helical decarlization lines are not observed in the texture under crossed polarized microscope. The dielectric spectroscopy is employed to observe the behavior of dielectric relaxation processes in these sample cells. A dielectric relaxation process is observed at a lower frequency than the Goldstone mode processes in DHFLC and FLC, which we call partially unwound helical mode (p-UHM). However, the p-UHM process is not observed in the sample cell in which the helical lines appear. The application of various amplitudes of probing ac voltages on this mode has shown the higher frequency shift, i.e., the larger the amplitude of ac voltage, the higher is the relaxation frequency of p-UHM. At sufficient amplitude of applied probing ac voltage, the p-UHM merges with the Goldstone mode process and is difficult to detect. However, the Goldstone mode relaxation frequency is almost independent of the cell geometry and sample configuration. The electro-optical behavior of the p-UHM has also been confirmed by electro-optical technique. The dielectric relaxation of UHM at a frequency lower than the Goldstone mode is interpreted as the fluctuation of partially unwound helix.
\end{abstract}

DOI: 10.1103/PhysRevE.95.062702

\section{INTRODUCTION}

Ferroelectric liquid crystals (FLCs) are important because of several aspects such as fast switching speed, high optical contrast, optical memory effect, and many more. The helical relaxation process in FLC materials is still a challenge for researchers [1]. The dynamics of helical unwinding process in FLC has been described either by the continuum theory [2] or by the theory where the helix is fractured into small segments [3]. Theoretically and experimentally, it was shown in DOBAMBC FLC material that the helical unwinding process is found to exceed and recede in accordance with theory close to the $\operatorname{Sm} C^{*}-\operatorname{Sm} A$ phase transition. The decrease in temperature of the FLC increases the rotational viscosity that seems to harden the unwinding process due to the motion of disclination lines [4]. The Glagarova et al. theoretical model suggests that disclination lines subsist in the vicinity of the upper and lower solid boundary of the sample cell structure [5]. Upon increasing the electric field strength, the upper and lower rows of the disclination approach each other and form the disclination in the form of a pair. If the electric field strength is further increased there is an increase in the domain due to the orientation of the director at the cost of the area of opponent domain having different director orientation. The dielectric relaxation of helical unwinding process for weak electric field can be estimated as $\tau=\gamma /\left(K q_{\mathrm{o}}{ }^{2}\right)$, where $\gamma$ is the rotational viscosity. $K$ and $q_{\mathrm{o}}$ are the elastic constant and helical wave vector of the FLC sample, respectively.

Kutnjac Urbanc et al. [6] have used the theoretical Landau model to study the excitation of the phase of tilt and relaxation of the helical process. It has been proposed that the total

\footnotetext{
*Corresponding author: abiradarnpl@gmail.com; abiradar@mail.nplindia.org
}

dielectric strength of helical FLC system is given by two separate processes: one is local variation of phase of tilt and the other is fluctuation of unwound helical structure. This theoretical analysis has shown that the relaxation of unwound helical structure as a whole has been observed at a lower frequency compared to the Goldstone mode frequency in a finite size of the sample. The finite size of the sample means the size of the electrode and particularly the length of the electrode along the helical axis. If the length of the system electrode is taken to several hundred times (not the integral number but in fraction so that the symmetry of the helix could be broken) the pitch of FLC, the relaxation peak of the unwound helical process is found at a lower frequency than the Goldstone mode, i.e., far away toward low frequency from the relaxation frequency peak of the Goldstone mode. But, if the length of the system electrode is reduced to about 5 times (not the integral number) the pitch of the material, then the relaxation process is found shifted toward the Goldstone mode peak.

Recently, two newly synthesized FLC materials have also shown a relaxation process at a frequency lower than the Goldstone mode attributed to a ferrielectric Goldstone-modelike process [7]. This process is obtained during the phase transition from ferroelectric to ferrielectric phase in the ferrielectric phase $\left(\mathrm{SmC}_{\gamma}^{*}\right)$ of antiferroeletric LC [8]. The helical dynamical process is a debatable research problem as it is demonstrated in selected FLC samples and needs more rigorous theoretical and experimental studies. However, this type of mode was also observed by our group earlier in some of the FLCs such as water-added FLC [9], pure FLC (KCFLC $7 \mathrm{~S}$ ), which becomes dominating when gold nanoparticles are added to it [10], and in $\mathrm{ZnO}$-added electroclinic liquid crystal [11]. All these measurements were performed at $500-\mathrm{mV}$ ac (alternating current) voltage of probing signal from an impedance analyzer. 
TABLE I. Parameters of DHFLC (LAHS-2) and FLC (SCE-13) materials at $25^{\circ} \mathrm{C}$.

\begin{tabular}{lccc}
\hline \hline $\begin{array}{l}\text { Material } \\
\text { name }\end{array}$ & $\begin{array}{c}\text { Spontaneous } \\
\text { polarization, } P_{\mathrm{s}} \\
\left(\mathrm{nC} / \mathrm{m}^{2}\right)\end{array}$ & $\begin{array}{c}\text { Helical pitch } \\
(\mu \mathrm{m})\end{array}$ & $\begin{array}{c}\text { Tilt angle, } \theta \\
\left({ }^{\circ}\right)\end{array}$ \\
\hline DHFLC (LAHS-2) & 45 & $\sim 0.3$ & 23 \\
FLC (SCE-13) & 25 & 10 & 21 \\
\hline \hline
\end{tabular}

In the present paper, the helical structure is unwound by the strong surface anchoring effect, i.e., strong rubbing of the polymide layer on the substrate. However, the thickness of the sample cell is still maintained more than the pitch value of the material but, due to strong anchoring, the decarlization lines are not visible. The dielectric spectroscopy and electrooptical studies have been carried out on sample cells of DHFLC (LAHS-2) [12] and conventional FLCs (SCE-13) at various amplitudes of the probing ac voltage and along with various $\mathrm{dc}$ bias voltages. The results have shown a noticeable relaxation peak at a frequency lower than the Goldstone mode. This low-frequency peak is found to shift toward higher frequency as the amplitude of ac voltage is increased and found to merge with the Goldstone mode at critical voltage. The lower-frequency process is attributed to the fluctuations of helix in partially unwound state close to the substrate, i.e.,

Cryst. $\stackrel{?}{\leftrightarrow} \mathrm{Sm} C^{*} \stackrel{58{ }^{\circ} \mathrm{C}}{\leftrightarrow} \mathrm{Sm} A \stackrel{62.5{ }^{\circ} \mathrm{C}}{\leftrightarrow}$ Iso. [DHFLC,LAHS - 2],

The FLC materials were introduced into the cells by means of capillary action at an elevated temperature, ensuring that the filling takes place in the isotropic phase. The electro-optical measurements of the FLC samples were done using a polarizing microscope (Axioskop-40, Germany) interfaced with a Canon digital camera and function/arbitrary wave-form generator (Agilent 33220A, USA). The dielectric measurements were carried out using a precision impedance analyzer (Wayne Kerr $6540 \mathrm{~A}, \mathrm{UK}$ ) in the frequency range $20 \mathrm{~Hz}$ to $1 \mathrm{MHz}$ at different amplitudes of probing ac and dc bias voltages.

\section{RESULTS AND DISCUSSION}

\section{A. Dielectric spectroscopy studies}

\section{Effect of amplitude of probing ac voltage on the relaxation process}

Figure 1 shows the real part of complex dielectric permittivity $\left(\varepsilon^{\prime}\right)$ and $\tan \delta$ curves at different amplitudes of probing ac voltage (ac electric voltage means the periodically varying sinusoidal applied voltage by the impedance analyzer used to measure the properties of the material under test; the maximum value of this voltage is $1 \mathrm{~V}$ ) at room temperature in DHFLC material. The sample cells are formed such that no helical lines are observed, i.e., the cell is made of strongly anchored substrates. The helix is partially unwound at the interface of substrate and FLC. Therefore, the helix is said to be partially unwound at the interface of substrate and bulk material. As partially unwound helical mode (p-UHM). This process is found to contribute a huge amount of permittivity along with the Goldstone mode in the ferroelectric phase $\left(\mathrm{SmC}^{*}\right)$. This is due to the fact that the partial unwinding state of the helix gives rise to the breaking of the director symmetry close to the substrate and FLC interface along the helical axis, resulting in the net spontaneous polarization which comes into effect at low amplitude of probing ac voltage.

\section{EXPERIMENTAL}

The sample cells for these studies were prepared using indium-tin-oxide-coated glass plates with a sheet resistance $\sim 20 \Omega / \square$ as the substrates. The planar alignment was obtained by treating the substrates first with adhesion promoter and then nylon (6/6). Thereafter, the substrates were rubbed unidirectionally along the desired direction on both glass plates. The thickness of the cells was maintained using an $8 \mu \mathrm{m}$ thick Mylar spacer for DHFLC and a $22 \mu \mathrm{m}$ thick spacer for FLC. Two types of ferroelectric liquid crystal materials, one DHFLC (LAHS-2) and the other FLC (SCE-13), were used for the present studies. DHFLC (LAHS-2) and FLC (SCE-13) have pitch values of 0.3 and $10 \mu \mathrm{m}$, respectively. The parameters of both the materials are given in Table 1.

Phase sequences of the two materials are seen in Fig. 1(a), the dielectric permittivity $\left(\varepsilon^{\prime}\right)$ remains almost independent of ac voltage up to $300 \mathrm{mV}$ and then starts increasing with the increase in the amplitude of ac voltage. The increase in permittivity from 50 to 200 is a huge jump at $1 \mathrm{~V}$ probing ac voltage. This increment cannot be due to the Goldstone mode process only because the Goldstone mode process is almost independent of the amplitude of ac voltage. This has also been confirmed in another sample cell in which the helical structure is formed as observed under crossed polarized microscope in transmission mode. There is no observable trace of the p-UHM process. Thus, the reason for this increment must be due to the existence of more than one dielectric process. Figure 1(b) shows the tan $\delta$ versus frequency plot at different amplitudes of probing ac voltage showing two different relaxation peaks. The relaxation frequency of the right-hand-side peak, which is independent of applied field, is due to a well-established Goldstone mode process (phase-fluctuation mode). The left-hand-side peak is highly dependent on ac voltage amplitude and shifts toward higher frequency with increase in the amplitude of applied ac voltage. As seen in Fig. 1(b), the Goldstone mode is seen at very low amplitude of applied ac voltage and the position of the peak remains almost the same with the increase in the amplitude of ac voltage. The amplitude of the dielectric permittivity is $\sim 50$ for lower ac voltage $(100 \mathrm{mV})$, which is purely due to phase-fluctuation mode, and it is understandable that the electric dipole moment needs a very low field for the fluctuations. As seen in Fig. 1(b), the position of the Goldstone mode peak appears at a higher frequency range 

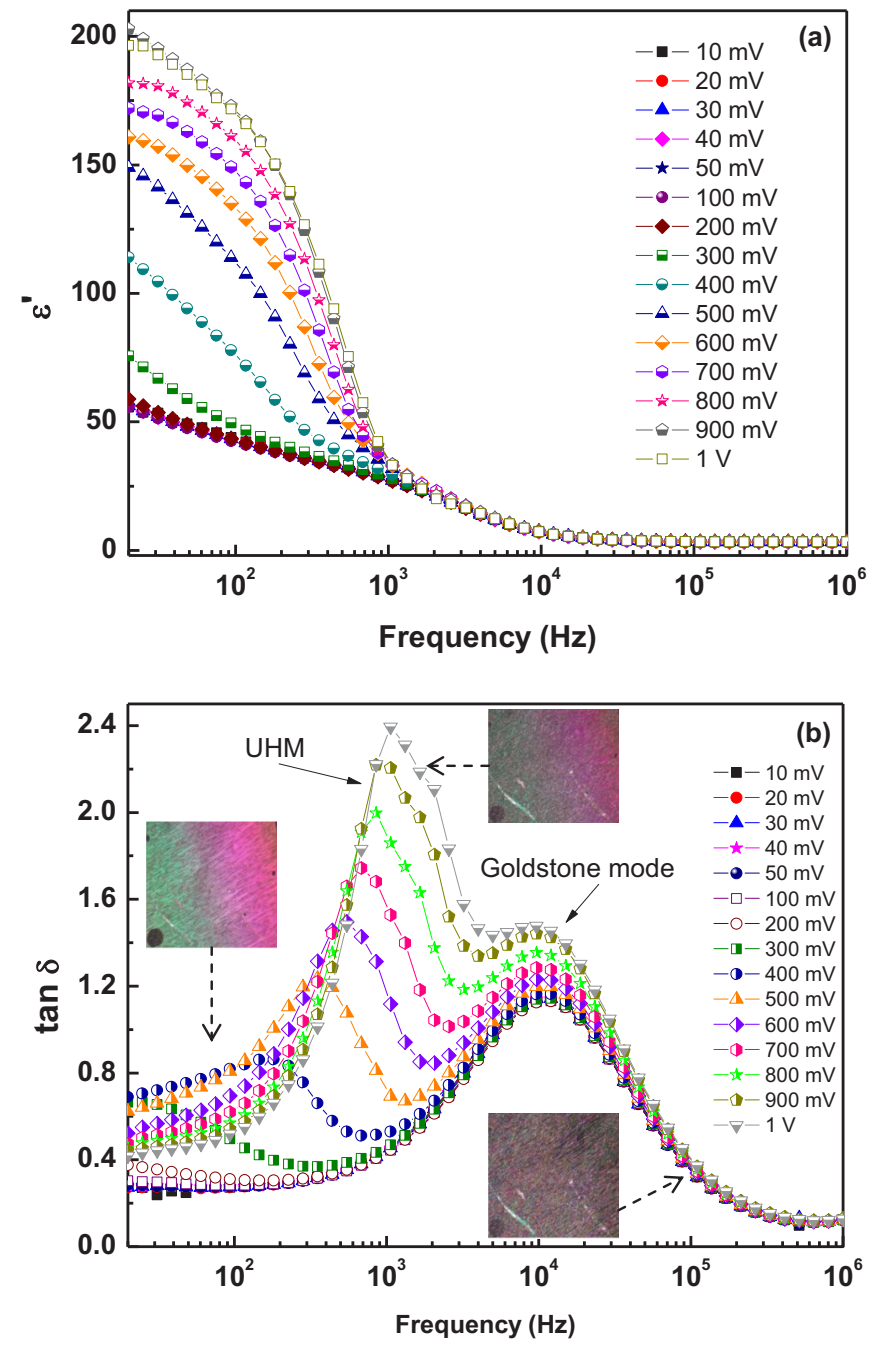

FIG. 1. (a) Real part of the complex dielectric permittivity versus frequency and (b) the $\tan \delta$ versus frequency at various amplitudes of probing ac voltage in DHFLC material. Insets of (b) show the behavior of optical micrographs of DHFLC at different frequencies during dielectric spectroscopy.

(more than $10 \mathrm{kHz}$ as observed from tan $\delta$ versus frequency curve) as compared to other conventional FLC materials (a few hundred and sometimes a few kilohertz) due to the fact that DHFLC materials have higher spontaneous polarization and viscosity than conventional FLC. The second peak in $\tan \delta$ starts appearing around 300-mV ac voltage, and its frequency and amplitude increase with the amplitude of applied voltage as seen in Fig. 1(b). The existence of partially unwound helix with net spontaneous polarization due to helical unwinding in the vicinity of the substrate and FLC interface is playing the significant role in the permittivity along with the Goldstone mode process. This mode can be called the partially unwound helical mode (p-UHM). Since the p-UHM peak appears at a critical voltage, therefore the UHM has threshold electric voltage and could vary from material to material depending upon sample cell conditions and material parameters, e.g., thickness of the cell, polarization, pitch, and viscosity of the FLC materials. Upon looking carefully, the peak amplitude of the $\mathrm{p}-\mathrm{UHM}$ process is larger than the Goldstone mode above
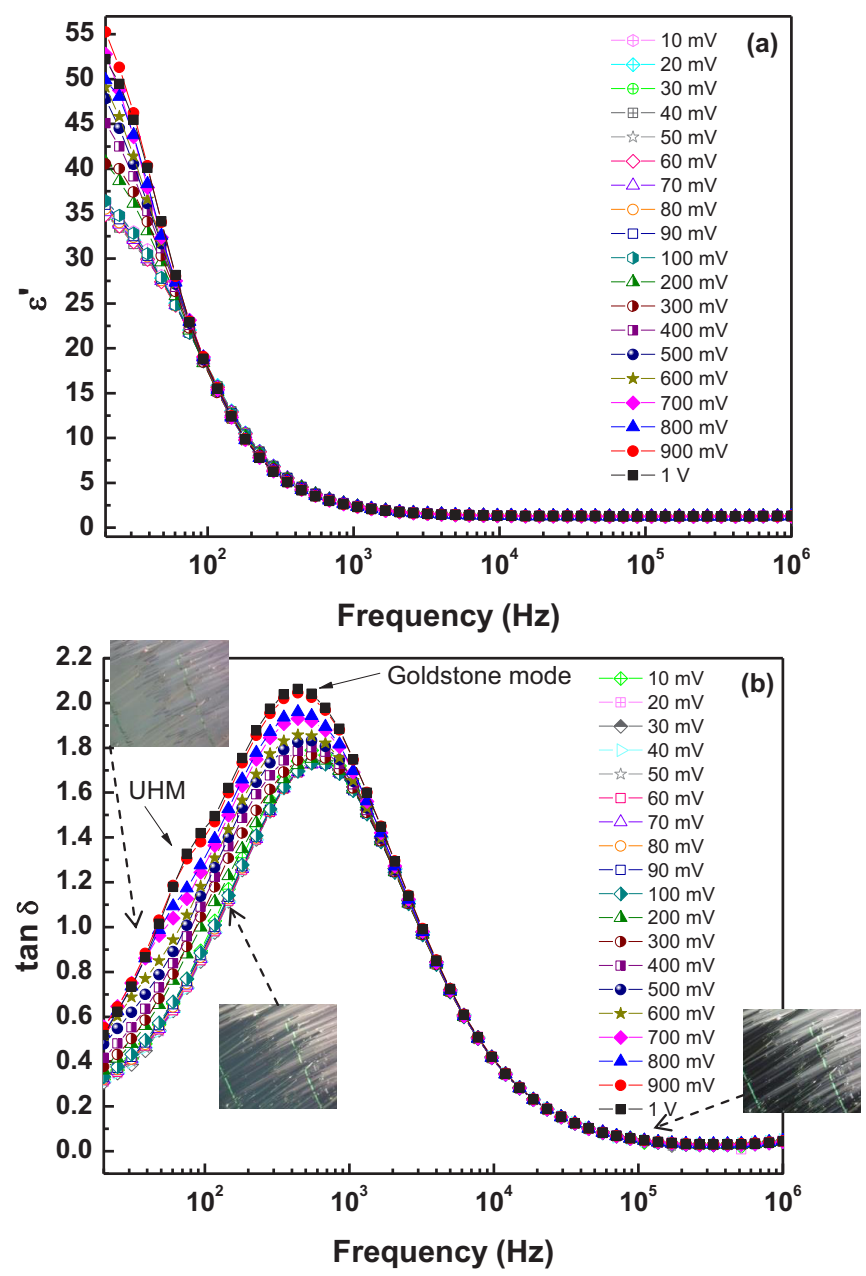

FIG. 2. (a) Real part of the complex dielectric permittivity versus frequency and (b) the $\tan \delta$ versus frequency as a function of amplitude of probing ac voltage in conventional FLC (SCE-13) material. Insets of (b) show the behavior of optical micrographs FLC at different frequencies during dielectric spectroscopy.

$600 \mathrm{mV}$, suggesting that most of the dielectric permittivity is due to the p-UHM, which is clearly reflected even in the dispersion curves also [Fig. 1(a)].

The p-UHM, as observed in the $\mathrm{SmC}^{*}$ phase only, seems not only characteristic of DHFLC materials but is also observed in conventional FLC (SCE-13) material. We have studied five FLC materials and all of them indicate the existence of p-UHM. However, the peak amplitude of the tan $\delta$ curve could vary from sample to sample of the same FLC material if the sample preparation conditions are not uniform. The data of a FLC (SCE-13) are presented in Fig. 2 at various amplitudes of probing ac voltages. The sample preparation parameters of the FLC sample are also almost the same as in DHFLC, i.e., anchoring energy and sample thickness are controlled in such a way that no complete helix formation could be observed due to strong substrate anchoring effect. The thickness of the sample cell is kept around $22 \mu \mathrm{m}$, which is more than the pitch value of the FLC material. As seen in the figures, the dielectric permittivity $\left(\varepsilon^{\prime}\right)$ remains static for lower amplitude of ac voltage levels (up to $100 \mathrm{mV}$ ) and then starts increasing with the increase in the ac voltages 


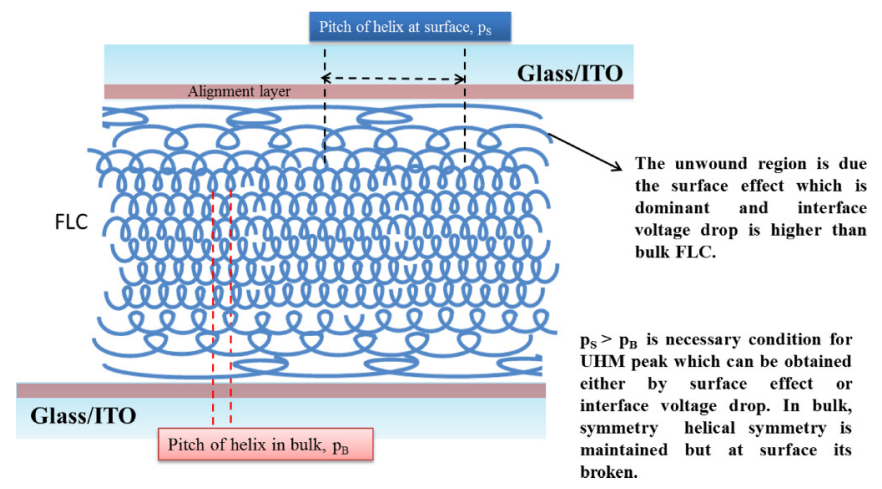

FIG. 3. Schematic of the helical unwinding at the interface of FLC/DHFLC and substrate. The depth (shaded region) of the unwinding could vary with the rubbing strength and amplitude of the applied electric field.

[Figs. 2(a) and 2(b)]. However, the increase in the permittivity is not as high as in the DHFLC material. The relaxation peak of UHM in the $\tan \delta$ versus frequency curves is not very clearly visible but an indication of a small hump is observed [Figs. 2(a) and 2(b)]. The reason could be understood in FLCs that the Goldstone mode relaxation frequency appears at low frequency at about $1 \mathrm{kHz}$ or in few hundred $\mathrm{Hz}$ ranges where the Goldstone mode process could be a dominant phenomenon. Secondly, the conditions for observing p-UHM are the suitable thickness of the cell alignment, anchoring energy, spontaneous polarization, and pitch value, which are very critical parameters. A clear p-UHM process could be observed after optimizing these parameters. The optimum thickness to observe the p-UHM process in DHFLC is $\sim 6-10 \mu \mathrm{m}$ thick, where tilt angle is $23^{\circ}$, pitch is $0.30 \mu \mathrm{m}$, and $P_{\mathrm{S}}$ is $45 \mathrm{nC} / \mathrm{cm}^{2}$. If the $\mathrm{p}$-UHM is not seen at room temperature due to variation in sample parameters or material properties, then it could be visualized at higher temperatures or even close to phase transition in the $\mathrm{SmC}^{*}$ phase. The temperature-dependent results are not presented here and will be published separately.

The most important parameters seem to be alignment (rubbing strength of the substrates) and thickness of the cell. This means that the partial unwinding of helical pitch is playing an important role in the appearance of this mode, as shown in the schematic diagram of the helix formation in a strongly anchored sample cell (Fig. 3). The model in the schematic describes the surface anchoring energy effect on the helical unwinding close to the substrate. The surface effect allows the helix to be partially unwound whereas in bulk it could remain unaffected. The pitch value of the FLC at interface of FLC and cell substrate could become several times larger than the bulk FLC, or even infinite. As the amplitude of ac voltage is increased along with the frequency scan, the surface unwinding gets into resonance with the bulk helix up to a certain depth of the sample and contributes to the net permittivity of the sample. After attaining a threshold voltage, the permittivity is found to decrease in some of the FLC materials (data of those materials are not presented and will be reported separately).

Even in FLC materials where the thickness is optimum $(\sim 22 \mu \mathrm{m})$ and alignment of FLC molecules is unidirectional, the p-UHM can be seen as shown in Fig. 2 in FLC material.

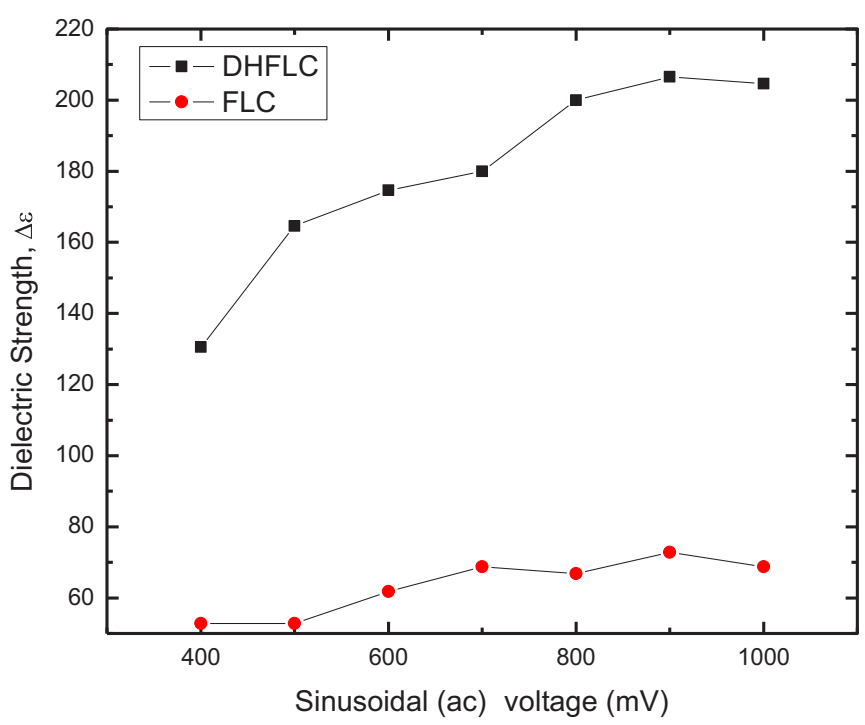

FIG. 4. Dielectric strength of p-UHM process of DHFLC (LAHS2) and FLC (SCE-13) as a function of amplitude of probing ac voltage.

In FLCs also at lower amplitude of ac voltages this mode is not seen but after crossing the threshold voltage $(500 \mathrm{mV})$ the relaxation peak starts appearing, and at $1 \mathrm{~V}$ the peak is seen and found shifting toward the Goldstone mode process [Fig. 2(b)].

The dielectric strength $\left(\Delta \varepsilon=\varepsilon_{\mathrm{o}}^{\prime}-\varepsilon_{\infty}^{\prime}\right)$ of two modes, where $\varepsilon_{o}^{\prime}$ and $\varepsilon_{\infty}^{\prime}$ are the static dielectric permittivity and dielectric permittivity at high frequency, respectively, is plotted against the amplitude of probing ac voltage in Fig. 4. As can be observed from Fig. 4, the amplitudes of dielectric strength of p-UHM for both the materials are found to increase upon increasing the amplitude of ac voltage, whereas the Goldstone mode is almost independent of the amplitude of ac voltage.

The relaxation frequency plotted in Fig. 5 is the frequency of maximum amplitude of the $\tan \delta$ peak of p-UHM, as marked in Figs. 1(b) and 2(b). The relaxation frequency of the p-UHM

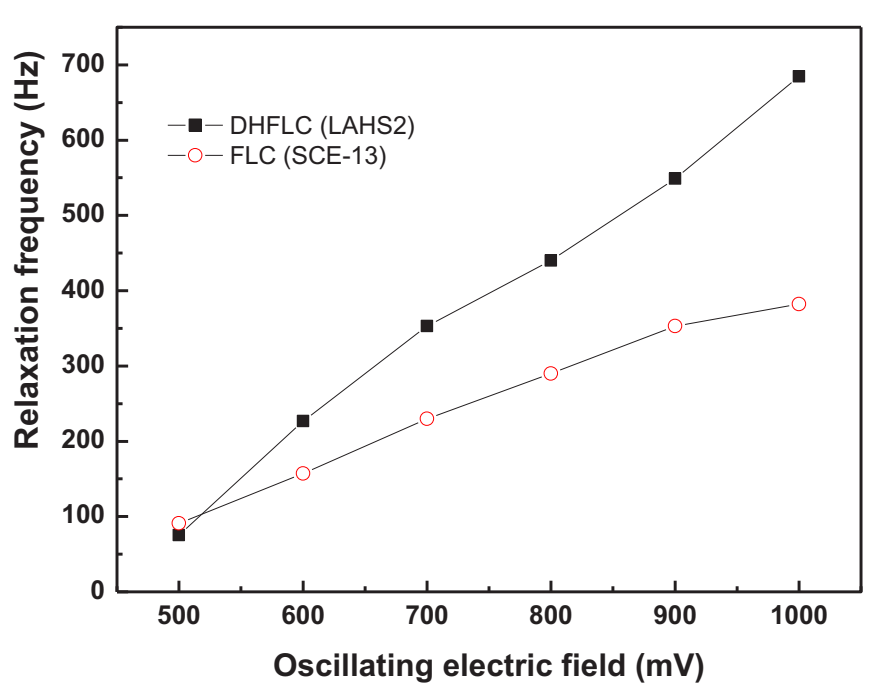

FIG. 5. Relaxation frequency of p-UHM versus amplitude of probing ac voltage of DHFLC (LAHS-2) and FLC (SCE-13). 
process increases linearly with the increase in the amplitude of ac voltage after crossing the threshold voltage, as seen in Fig. 5 in DHFLC material and FLC materials. This means that below threshold field the contribution to the dielectric permittivity is only due to the Goldstone mode; afterward, at higher ac voltages, it is due to both p-UHM and phason fluctuation Goldstone modes.

The increase in the relaxation frequency is also clearly reflected in the FLC material, as seen in Fig. 5. The p-UHM relaxation frequency which appears before the Goldstone mode is not limited to the deformed helix FLC material only but an inherent characteristic of the $\mathrm{SmC}{ }^{*}$ phase of FLC-class materials and must be related to the partially unwound helical structure of the material. This is due to the fact that for lower ac voltage level (below $100 \mathrm{mV}$ ) the helical structure in bulk (Fig. 5) is unaffected, and when applied voltage crosses the threshold voltage, the structure gets partially distorted and responds to the applied field, contributing to the dielectric permittivity. The relaxation frequency and amplitude of $\mathrm{p}$-UHM in the $\tan \delta$ curve increase with the ac voltage [Figs. 1(b), 2(b), and 5] and finally merge with the Goldstone mode. It is worth mentioning here that it is not necessary to have a complete helical structure in the cell to observe this mode. This could be realized even in the twisted helical structure as shown in Fig. 5, where the pitch value of the FLC is $\sim 10 \mu \mathrm{m}$ and the thickness of the cell is $8 \mu \mathrm{m}$. Kutnjac-Urbanc et al. predicted such type of mode theoretically as early as 1995 but so far the concept is lacking experimental exploration with clear evidence of the existence of this mode [6]. In the present study, the systematic measurements have thrown light on the qualitative analysis of the existence of such type of helical process.

\section{Effect of bias voltage on the dielectric relaxation process}

In order to analyze the dc bias-dependent behavior of dielectric properties of DHFLC material, the various dc bias voltages are applied in two particular cases of ac voltages, i.e., at $100 \mathrm{mV}$ and at $1 \mathrm{~V}$. The data are presented for DHFLC material only because the behavior is the same in FLC also. Figure 6 shows the real part of complex dielectric permittivity, $\varepsilon^{\prime}$ and $\tan \delta$ curves of the dielectric process at low ac voltage of $100 \mathrm{mV}$ for different dc bias voltages at room temperature in DHFLC material. It is clear from Fig. 6(a) that the dielectric permittivity decreases continuously with the increase in the bias voltage, which is a well-known behavior of the Goldstone mode process in FLC [13]. It is interesting to see that in the $\tan \delta$ versus frequency curve for lower probing ac voltage there is no trace of $\mathrm{p}$-UHM process on the left side of the Goldstone mode peak frequency, suggesting that it is a pure Goldstone mode process [Fig. 6(b)]. Upon increasing the amplitude of probing ac voltage to $\sim 1 \mathrm{~V}$ the dielectric permittivity jumps to almost 3 times, as seen in Fig. 7(a), and two relaxation processes appear in the $\tan \delta$ versus frequency curve. As the dc bias voltage is increased, both the processes get suppressed to low amplitude. However for higher dc bias voltages more than $2 \mathrm{~V}$, the p-UHM gets suppressed more than the Goldstone mode [Fig. 7(b)], suggesting that the partially unwound helix goes in a further unwinding state. It is important to mention here that most of the contribution to the dielectric permittivity in the
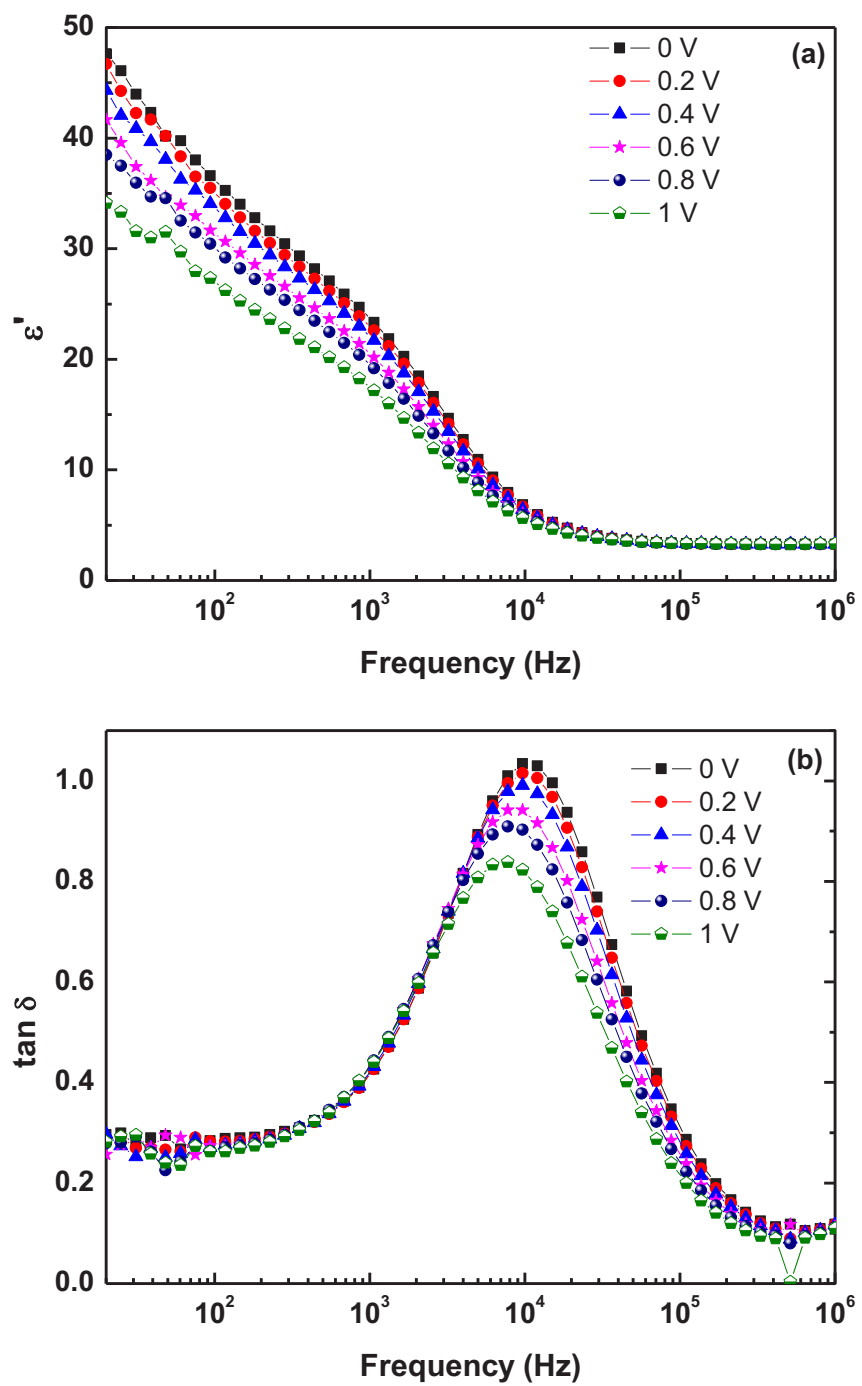

FIG. 6. (a) Real part of the complex dielectric permittivity versus frequency and (b) $\tan \delta$ versus frequency at various bias electric voltages. The amplitude of probing ac voltage is kept at $100 \mathrm{mV}$ in DHFLC (LAHS-2) material.

$\mathrm{Sm}^{*}$ phase of DHFLC material at higher ac voltage is due to partially unwound helical fluctuation mode. Other important information is that the relaxation frequency of $\mathrm{p}$-UHM is observed independently of bias field (Fig. 7) like the Goldstone mode but linearly dependent on ac field (Fig. 5).

\section{Electro-optical studies}

a. Frequency-dependent electro-optical studies. As it is well known, the application of electric field applies the torque on the FLC molecules, which changes the orientation of molecular director. Therefore, it is supposed that the application of ac and dc voltages on the sample under crossed optical microscope could give more information about the molecular modes by observing the optical micrographs [as inset micrographs of Figs. 1(b) and 2(b)]. The optical micrographs of DHFLC sample have been monitored under crossed polarized microscope at different frequencies during the performance of dielectric spectroscopy, as shown in the 

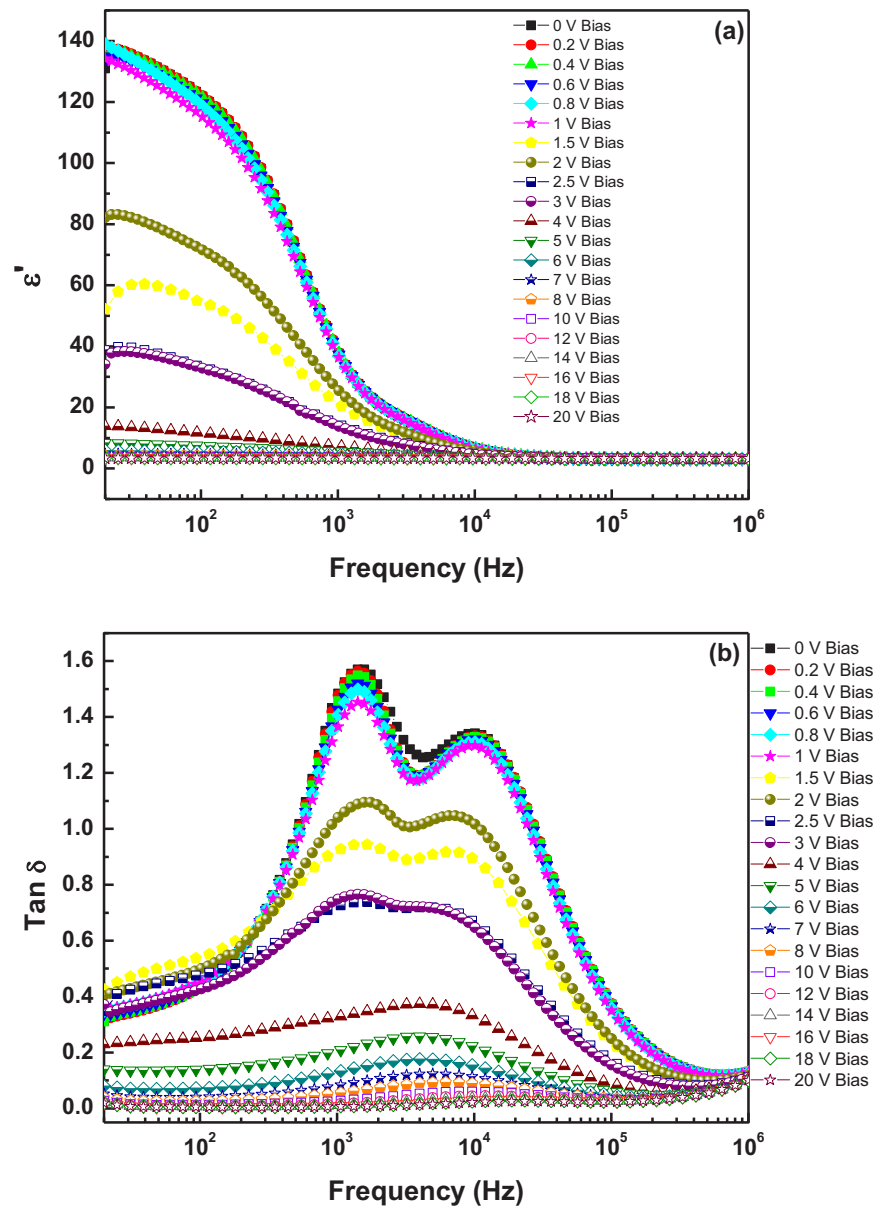

FIG. 7. (a) Real part of the complex dielectric permittivity versus frequency and (b) $\tan \delta$ versus frequency at various bias electric voltages. The amplitude of probing ac voltage is kept at $1 \mathrm{~V}$ in DHFLC (LAHS-2) material.

insets of Figs. 1(b) and 2(b). The frequencies of capturing the textures during the dielectric spectroscopy scan are $100 \mathrm{~Hz}$ and $100 \mathrm{kHz}$. The $100-\mathrm{Hz}$ frequency is selected because at this frequency the contribution of partially unwound helical mode to dielectric permittivity is seen as described in previous discussions [Figs. 1(a) and 2(a)]. The $100 \mathrm{kHz}$ is that frequency where there is no contribution of p-UHM or the Goldstone mode (Figs. 1 and 2). The confirmation of the change in optical micrographs due to helical fluctuation mode is observed under crossed polarizers.

Figure 8 reveals the observation of the micrograph of DHFLC material at $100 \mathrm{~Hz}$ and $100-\mathrm{kHz}$ frequency at $100-\mathrm{mV}$ amplitude of ac voltage with no bias. As can be seen from Figs. 8(a) and 8(b), there is no change in the optical micrographs at these two frequencies. The dielectric measurement data corresponding to this observation are shown in Fig. 1 for DHFLC and confirm no appearance of p-UHM at $100 \mathrm{mV}$. Upon increasing the amplitude of probing ac voltage to $1 \mathrm{~V}$ with no bias field, the results are as shown in Fig. 9. There is a clear change in light transmission intensity having the same optical texture [Fig. 9(a)] at 100-Hz frequency in comparison to the optical micrograph in Fig. 8(a). The applied frequency is below the observed relaxation frequency of the p-UHM
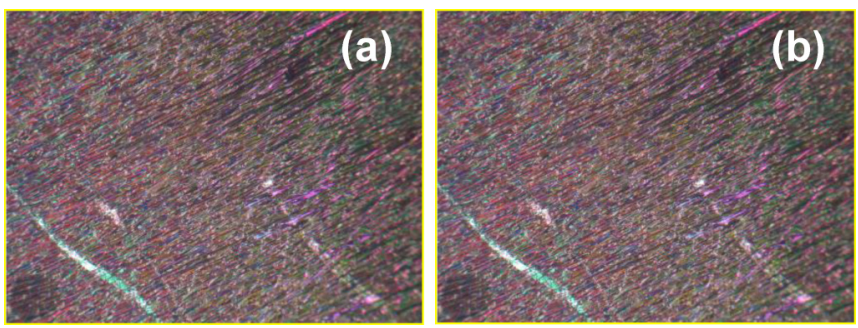

FIG. 8. Optical micrographs of DHFLC (LAHS-2) under crossed polarized microscope at fixed $100-\mathrm{mV}$ ac voltage at (a) frequency $100 \mathrm{~Hz}$ and (b) at $100 \mathrm{kHz}$.

process of DHFLC. At the $1 \mathrm{kHz}$ frequency, which is the relaxation frequency of the UHM, the change in transmission intensity of optical micrograph is found to be decreasing but is not exactly the intensity of the scattered state, i.e., contribution of p-UHM is still there [Fig. 9(b)]. The optical micrograph at $100-\mathrm{kHz}$ frequency [Fig. 9(c)] is observed almost in the scattered state, as can be confirmed from Figs. 9(c) and 8(b). The study suggests that whenever there is a change in the transmission intensity of the micrographs, the $\mathrm{p}$-UHM process appears but there is no change in the texture of the material. The dielectric spectra corresponding to this observation can be seen in Figs. 1(a) and 1(b). In dielectric spectra also, there is no contribution from any mode at high frequency, $100 \mathrm{kHz}$.

The study has been extended to conventional FLCs and the optical micrograph observations are given in Fig. 10. The same technique has been adopted for the optical observations of FLCs as in the case of DHFLC. Figure 10(a) shows the texture of the sample just before the measurement with no voltage application. The capture frequencies of the micrograph are $50 \mathrm{~Hz}, 221 \mathrm{~Hz}$ (relaxation frequency of p-UHM), and $100 \mathrm{kHz}$. The amplitude of probing ac voltage is kept at $1 \mathrm{~V}$. In Fig. 10(b), a clear change in the light transmission intensity has been observed at the frequency of $50 \mathrm{~Hz}$. The frequency of $50 \mathrm{~Hz}$ is sufficiently below the relaxation frequency of $221 \mathrm{~Hz}$ of p-UHM in FLC. The dielectric permittivity is also high enough at $50 \mathrm{~Hz}$. The texture of the optical micrograph at $221 \mathrm{~Hz}$ (approximate relaxation frequency of p-UHM) [Fig. 10(c)] lies, according to transmission intensity, in between the micrographs at $50 \mathrm{~Hz}$ and $100 \mathrm{kHz}$. Then, at higher frequency, i.e., $100 \mathrm{kHz}$ [Figs. 10(d) and 2(b)], the micrograph resembles the micrograph with no electric field [Fig. 10(a)]. This means that the molecules are showing neither the helical fluctuation nor molecular switching corresponding to the applied field. In all these observations of DHFLCs and FLCs, the changes in optical micrographs have been observed
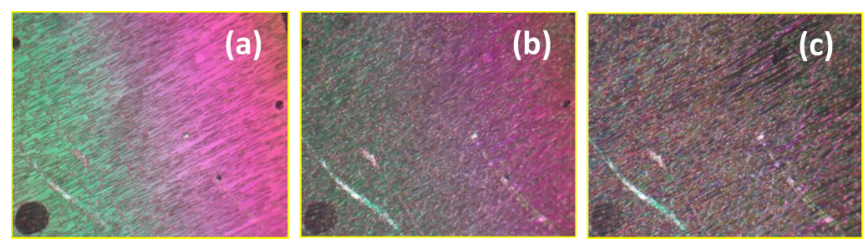

FIG. 9. Optical micrographs of DHFLC (LAHS-2) under crossed polarized microscope at fixed $1-\mathrm{V}$ ac voltage at (a) frequency $100 \mathrm{~Hz}$, (b) at $1 \mathrm{kHz}$, and (c) at $100 \mathrm{kHz}$. 

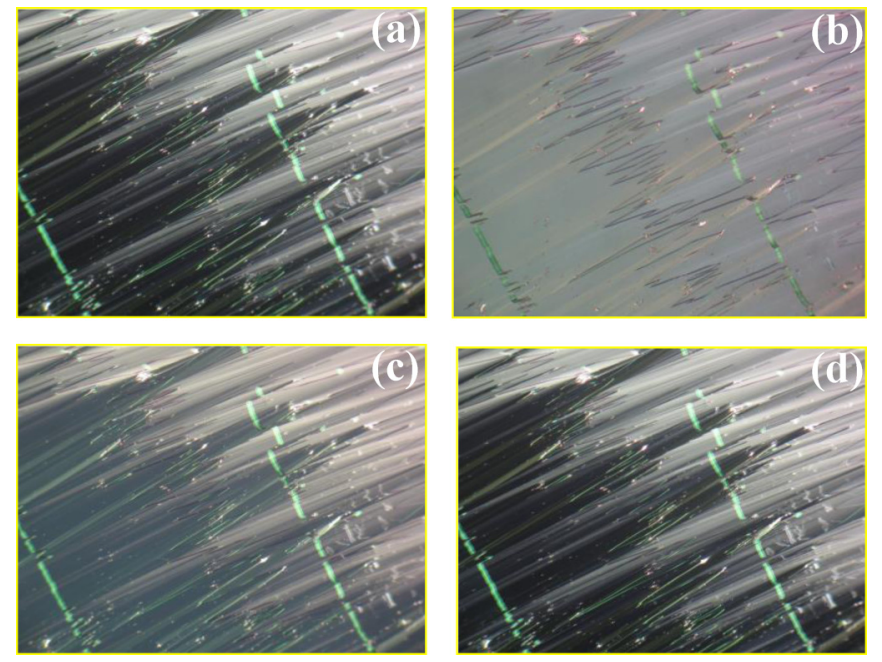

FIG. 10. Optical micrographs of FLC (SCE-13) at fixed amplitude of $1-\mathrm{V}$ ac voltage (a) before measurement, (b) at $50 \mathrm{~Hz}$, (c) at $221 \mathrm{~Hz}$, and (d) at $100 \mathrm{kHz}$.

only below the relaxation frequency of p-UHM and not just below the Goldstone mode process. This confirms that the change in the optical transmission intensity of micrograph is due to the $\mathrm{p}-\mathrm{UHM}$ process and not because of the Goldstone mode.

b. Dependence of electro-optical on amplitude of probing ac voltage. So far the frequency dependence of the optical texture has been carried out with constant amplitude of probing ac voltage. In the following section the frequency has been kept constant and the amplitude of probing ac signal is varied for the study of the optical micrographs of DHFLC and conventional FLC.

The 100-Hz frequency, applied to DHFLC, is sufficiently below the relaxation frequency of the p-UHM as discussed in the previous section. Figures 11(a)-11(f) exhibit the optical micrographs of DHFLC at different amplitudes of ac voltages and at a fixed 100-Hz frequency. At amplitudes of $500 \mathrm{mV}$ and $1 \mathrm{~V}$ of applied ac signal from arbitrary wave-form generator, the function generator is used because there is a limitation of maximum $1 \mathrm{~V}$ in the impedance analyzer. The textures of optical micrographs are almost the same
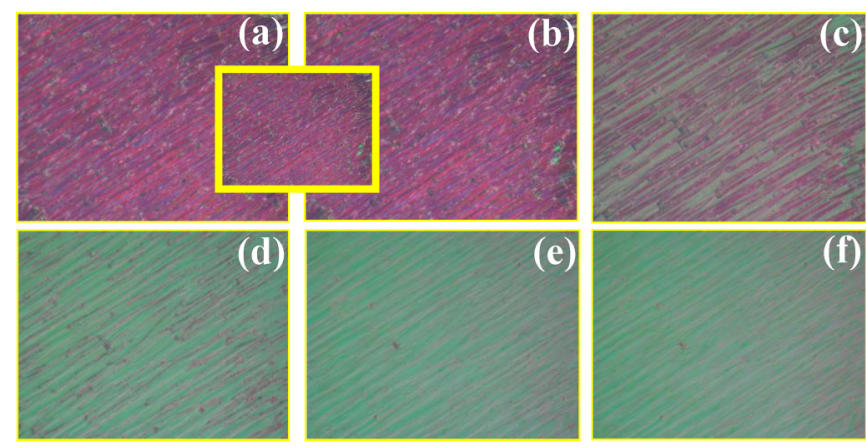

FIG. 11. Optical micrographs of DHFLC (LAHS-2) at fixed frequency $100 \mathrm{~Hz}$ of ac signal and at (a) $500-\mathrm{mV}$, (b) $1-\mathrm{V}$, (c) $2-\mathrm{V}$, (d) 4-V, (e) 6-V, and (f) 8-V ac voltage. Inset of (a) and (b) shows the optical micrographs of material before applying any field.
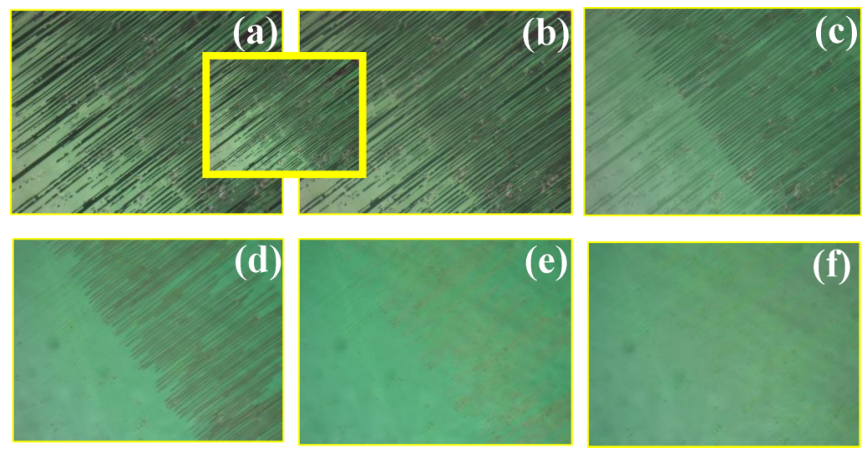

FIG. 12. Optical micrographs of FLC (SCE-13) at fixed frequency $100 \mathrm{~Hz}$ of ac voltage and at amplitude of (a) $500-\mathrm{mV}$, (b) $1-\mathrm{V}$, (c) $2-\mathrm{V}$, (d) $4-\mathrm{V}$, (f) 6-V, and (e) $8-\mathrm{V}$ ac voltage. Inset of (a) and (b) shows the micrograph of sample before applying any voltage.

except for the change in transmission intensity of domains. However, upon increasing the level of ac voltage, there is a change in color of the micrograph and texture along with the change in size of domains as well. This observation confirms that the molecular orientation starts beyond the threshold voltage $\sim 1 \mathrm{~V}$. Below the threshold amplitude of ac voltage, the possibilities of molecular orientation are ruled out and the change in micrographs is due to the p-UHM only. Hence, the mere change in optical micrographs at various electrical parameters (amplitude of ac voltage and dc bias voltage) is observed due to the partially unwound helical structure fluctuations.

The same optical concept is also applied to a FLC sample. Figures 12(a)-12(f) show the observation of optical micrographs of a FLC sample. As can be seen in Fig. 12, the only difference between the inset micrograph and Figs. 12(a) and 12 (b) is the difference in the intensity and not in the domain size and color change. Upon increasing the voltage [Figs. 12(c)-12(f)], there is a change in the in domain size and color of the micrograph. These observations confirm the existence of the p-UHM process and again rule out the possibility of any other mode at $1-\mathrm{V}$ amplitude of ac signal and low frequencies.

\section{CONCLUSIONS}

In summary, the helical relaxation dynamics of DHFLC and FLC have been experimentally observed under the partially unwound helical state by the surface anchoring effect. The theoretical observation has predicted such types of mode in FLCs materials. The dielectric spectroscopy reveals a relaxation process at a frequency lower than the relaxation frequency of the well-established Goldstone mode process. The dielectric strength and relaxation frequency of this mode are appreciably dependent on the probing ac voltage and found to increase with the increase in the probing ac voltage. Both the dielectric modes are dc bias voltage dependent; however, the observed mode could be completely suppressed at a lower field than the Goldstone mode. The dependency of dielectric strength and relaxation frequency points toward the partially unwinding state of the helix close to the anchored surface of the cell and is called the partially unwound helical mode (p-UHM). 
The p-UHM process has also been studied by electro-optical method and it is observed that whenever the p-UHM process occurs, there is a change in the transmission intensity of the micrograph without affecting the texture of the sample.

\section{ACKNOWLEDGMENTS}

We sincerely thank the Director, National Physical Laboratory, New Delhi for his continuous encouragement in this work. We would like to thank the Department of Science and
Technology, New Delhi, India for financial support through Project Grant GAP-150632 at NPL, New Delhi, India. We sincerely thank Dr. A. K. Thakur of Central University of Jammu, India for useful discussion, and Professor W. Haase and Dr. A. V. Lapanik of Technical University Darmstadt, Germany for providing the DHFLC (LAHS-2) material. One of the authors (A.M.B.) is thankful to Council of Scientific and Industrial Research (CSIR, India) for the financial assistance under an Emeritus Scheme.
[1] L. A. Beresnev, L. M. Blinov, M. A. Osipov, and S. A. Pikin, Ferroelectric Liquid Crystals (Gordon and Breach Science, Philadelphia, PA, 1988).

[2] R. B. Meyer, Mol. Cryst. Liq. Cryst. 40, 33 (1977); Appl. Phys. Lett. 12, 281 (1968).

[3] J. K. Song, U. Manna, and J. K. Vij, Europhys. Lett. 82, 26003 (2008).

[4] K. Yoshino, Y. Iwasaki, T. Uemoto, and Y. Inuishi, Jpn. J. Appl. Phys. 18(Suppl. 18-1), 427 (1979).

[5] M. Glagarova, J. Fousek, L. Lejcek, and J. Pavel, Ferroelectrics 58, 161 (1984).

[6] B. Kutnjac-Urbanc and B. Žekš, Phys. Rev. E 52, 3892 (1995).
[7] L. C. Huang and C. M. Fu, Acta Phys. Pol., A 129, 97 (2016).

[8] S. Hiller, S. A. Pikin, W. Haase, J. W. Goodby, and I. Nishiyama, Jpn. J. Appl. Phys. 33, L1096 (1994).

[9] G. Singh, A. Choudhary, G. Vijaya Prakash, and A. M. Biradar, Phys. Rev. E 81, 051707 (2010).

[10] A. Kumar, J. Prakash, and A. M. Biradar, Liq. Cryst. 37, 247 (2010).

[11] A. Malik, A. Choudhary, P. Silotia, and A. M. Biradar, J. Appl. Phys. 110, 064111 (2011).

[12] F. V. Podgornov, A. M. Suvorova, A. V. Lapanik, and W. Haase, Chem. Phys. Lett. 479, 206 (2009).

[13] S. Kaur, A. K. Thakur, S. S. Bawa, and A. M. Biradar, Physica B 344, 133 (2004). 